\title{
Polycrystalline solidification in a quenched 2D colloidal system
}

\author{
P Dillmann, G Maret and P Keim \\ Fachbereich Physik, Universität Konstanz, D-78457 Konstanz, Germany \\ E-mail: peter.keim@uni-konstanz.de
}

\begin{abstract}
We investigate a two-dimensional colloidal system when quenched from the isotropic fluid phase into the crystalline phase. In thermal equilibrium one finds two continuous phase transitions with a hexatic phase in between the fluid and the crystalline phase. Here, no indications of this anisotropic fluid are observed if the system is driven out of equilibrium. Therefore we investigate whether a nucleation process may describe the scenario. To identify the crystallites a criterion based on the local bond-order field is introduced. With this at hand the growth of the crystallites is investigated. At early times, small crystallites start to grow in the supercooled isotropic phase and later, when the crystallites start to touch each other, the small ones are merged into the bigger crystallites due to a ripening process.
\end{abstract}

\section{Introduction}

How a two-dimensional crystal melts has been a matter of debate over decades. Kosterlitz and Thouless [1, 2], for example, predicted a continuous transition induced by topological defects whereas first-order transitions mediated by grain boundaries or geometrical defects were postulated by Chui [3] and Glaser and Clark [4], respectively. The existence of a hexatic phase was predicted by Nelson and Halperin [5, 6] as an intermediate phase between the isotropic liquid and crystalline phase with two continuous defectmediated transitions. As colloids can be easily visualized by video microscopy, this prediction has been verified in such 2D colloidal systems [7-11]. In [12, 13] the mechanism of melting was determined on a microscopic level. Indeed the transitions were identified to be continuous and no hysteresis of the transition points was found in melting and cooling cycles of the system, keeping it always in thermal equilibrium. In this paper we address the question of what happens if the system is cooled down very rapidly. Will the hexatic phase be observable when the system is quenched out of equilibrium from the isotropic fluid to the crystalline phase?

\section{Experimental system}

Our system consists of spherical super-paramagnetic colloidal particles with diameters $d=4.5 \mu \mathrm{m}$ immersed in water.
The particles are confined by gravity to the water/air interface formed by a droplet which is suspended by surface tension in a top-sealed cylindrical hole (6 mm diameter) of a glass plate. A magnetic field $\vec{H}$ is applied perpendicular to the water/air interface inducing a magnetic moment $\vec{M}=\chi \vec{H}$ in each particle, where $\chi$ is the effective susceptibility per bead. This leads to a repulsive dipole-dipole pair interaction $E_{\text {mag }}$ with the dimensionless interaction strength $\Gamma$ given by the ratio of the potential magnetic energy versus thermal energy:

$$
\Gamma=\begin{aligned}
& E_{\mathrm{mag}} \\
& k_{\mathrm{B}} T
\end{aligned}=\begin{aligned}
& \mu_{0} \chi^{2} H^{2} \\
& 4 \pi k_{\mathrm{B}} T
\end{aligned}(\pi \rho)^{3 / 2} .
$$

Here $\rho$ is the $2 \mathrm{D}$ particle density and the average particle distance is given by $a=1 / \sqrt{ } \rho$. The interaction strength is externally controlled by means of the magnetic field $\vec{H}$; it can be interpreted as an inverse temperature and controls the phase behaviour of the system with a transition temperature from isotropic fluid to hexatic $\Gamma_{\mathrm{i}} \approx 57.5$ and a melting temperature of hexatic to crystalline of $\Gamma_{\mathrm{m}} \approx 60[12,13]$. The ensemble of particles is visualized by video microscopy with a CCD 8-bit greyscale camera $(1392 \times 1040$ pixel $)$ and the field of view has a size of $1158 \times 865 \mu \mathrm{m}^{2}$ containing 6800 particles whereas the whole sample is roughly 30 times larger. The images are captured at a frame rate of $2 \mathrm{~Hz}$ and they are analysed in situ. One single particle contains 65 pixels and the resolution of the barycentre of each particle is about $50 \mathrm{~nm}$. Digital image 


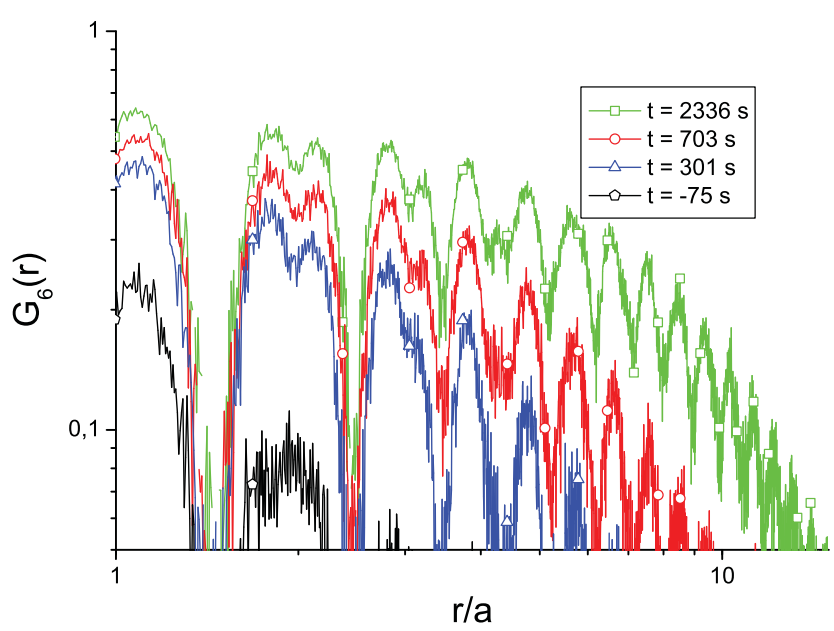

Figure 1. $\log -\log$ plot of the orientational correlation function for different time steps before and after the quench from $\Gamma=20$ to 80 . Before the quench the correlation decays within a few lattice sites, while after the quench it increases with time. We never observed an algebraic decay (linear curve in the log-log plot) which would be the signature of the hexatic phase.

analysis provides size, number and position of the colloids at each time step and the trajectories of the particles are recorded.

Starting from the liquid state we can induce the crystallization process by increasing the external magnetic field $\vec{H}$ and therefore decreasing the system temperature below the liquid-solid phase transition temperature. The cooling rate is only limited by the electronics of the power supply and is measured to have values about $\mathrm{d} \Gamma / \mathrm{d} t \approx 10^{4} \mathrm{~s}^{-1}$ in our system.

\section{Long-range order}

To investigate whether a signature of the hexatic phase occurs in the non-equilibrium situation, we calculated the bond-order correlation function

$$
G_{6}(r)=\left\langle\left|\psi\left(\overrightarrow{r_{k}}\right) \psi^{*}\left(\vec{r}_{j}\right)\right|\right\rangle_{k j}=\left\langle\left|\psi(\vec{r}) \psi^{*}(\overrightarrow{0})\right|\right\rangle,
$$

where the brackets denote the ensemble average taken over all $N(N-1) / 2$ particle-pair distances $r=\left|\vec{r}_{k}-\vec{r}_{j}\right|$ for each configuration with $N$ particles in the field of view. A quasilong-range decay of the orientational correlation $G_{6}(r) \sim r^{-\eta_{6}}$ corresponds to the hexatic phase (with $\eta_{6}<1 / 4$ ) $[5,6]$. Exponential decay is found in the isotropic phase $G_{6}(r) \sim$ $\mathrm{e}^{-r / \xi_{6}}$ and in the monocrystalline phase the correlation is perfect long-range $\lim _{r \rightarrow \infty} G_{6}(r) \neq 0$ [13]. In figure 1 the correlation is plotted for four different time steps. Before the quench one finds exponential decay and also after the quench the orientational correlation is always short range. Roughly $1700 \mathrm{~s}$ after the quench a linear decay fits better (or almost as good as the exponential one), maybe indicating the polycrystalline structure. An algebraic decay was always found to fit the data worse. Therefore no indication of the hexatic phase is observed, if the system is driven out of equilibrium from the isotropic fluid into the crystalline phase.

\section{Local bond-order field}

Since quasi-long-range orientational order does not develop during the solidification one has to investigate the degree of order locally. In order to identify particles with local crystalline order we investigated different methods using Voronoi construction and a bond-order parameter.

The first is based on the shape factor $\zeta_{i}=C_{i}^{2} / 4 \pi S_{i}$ which relates the circumference $C_{i}$ of the Voronoi cell of particle $i$ to its area $S_{i}[16,17]$. This quantity is minimized for circles $\left(\zeta_{\text {circle }}=1\right)$ and has typical values for a perfect pentagon $\left(\zeta_{\text {pentagon }}=1.156\right)$, a hexagon $\left(\zeta_{\text {hexagon }}=1.103\right)$ or a heptagon $\left(\zeta_{\text {heptagon }}=1.073\right)$. Figure 2 (left) shows the probability distribution of $\zeta$ for a quenched system shortly after the quench. Two weak minima are observable, dividing the distribution in three regions according to the five-, six-and sevenfold particles. But when the probability distributions are plotted separately for those differently coordinated particles, one observes a strong overlap of the curves at the minima [19] and one can hardly introduce a threshold. Therefore this criterion is not sufficient to distinguish locally between crystallike and fluid-like particles.
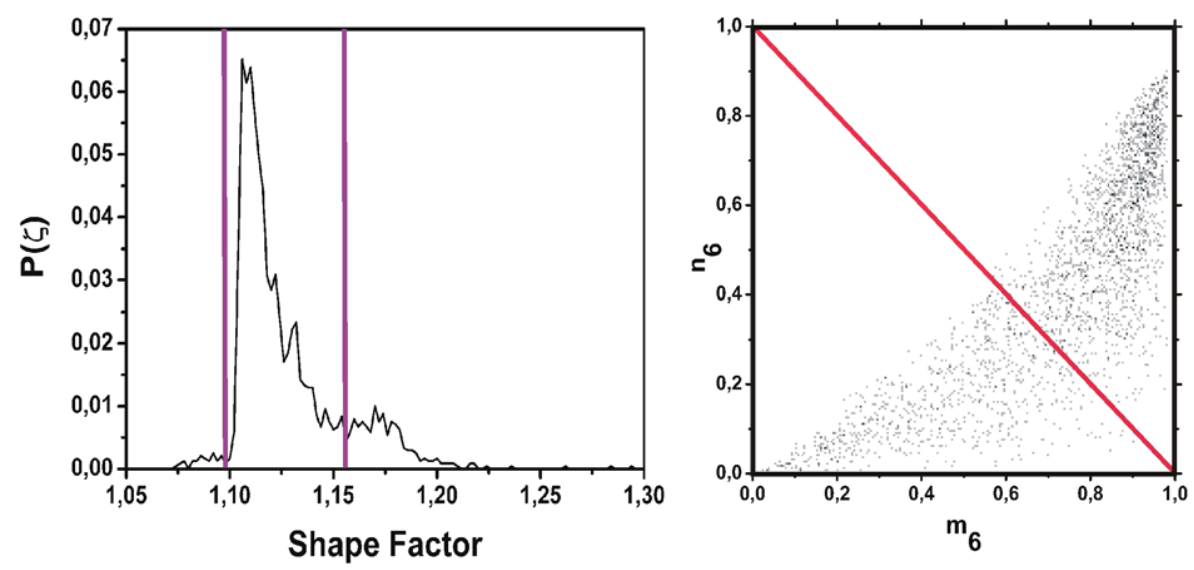

Figure 2. Left: probability distribution of the shape factor of a system $198 \mathrm{~s}$ after the quench from $\Gamma=20$ to 100 . The distribution can be divided into three regions, but a strong overlap of the individual distributions of five-, six- and sevenfold particles is underlying. Right: greyscale image $(\mathrm{au})$ of the probability distribution of the magnitude of the local bond-order parameter $m_{6}$ versus the magnitude of the projection of $\psi_{k}$ to the mean of the nearest neighbours $n_{6}$. 

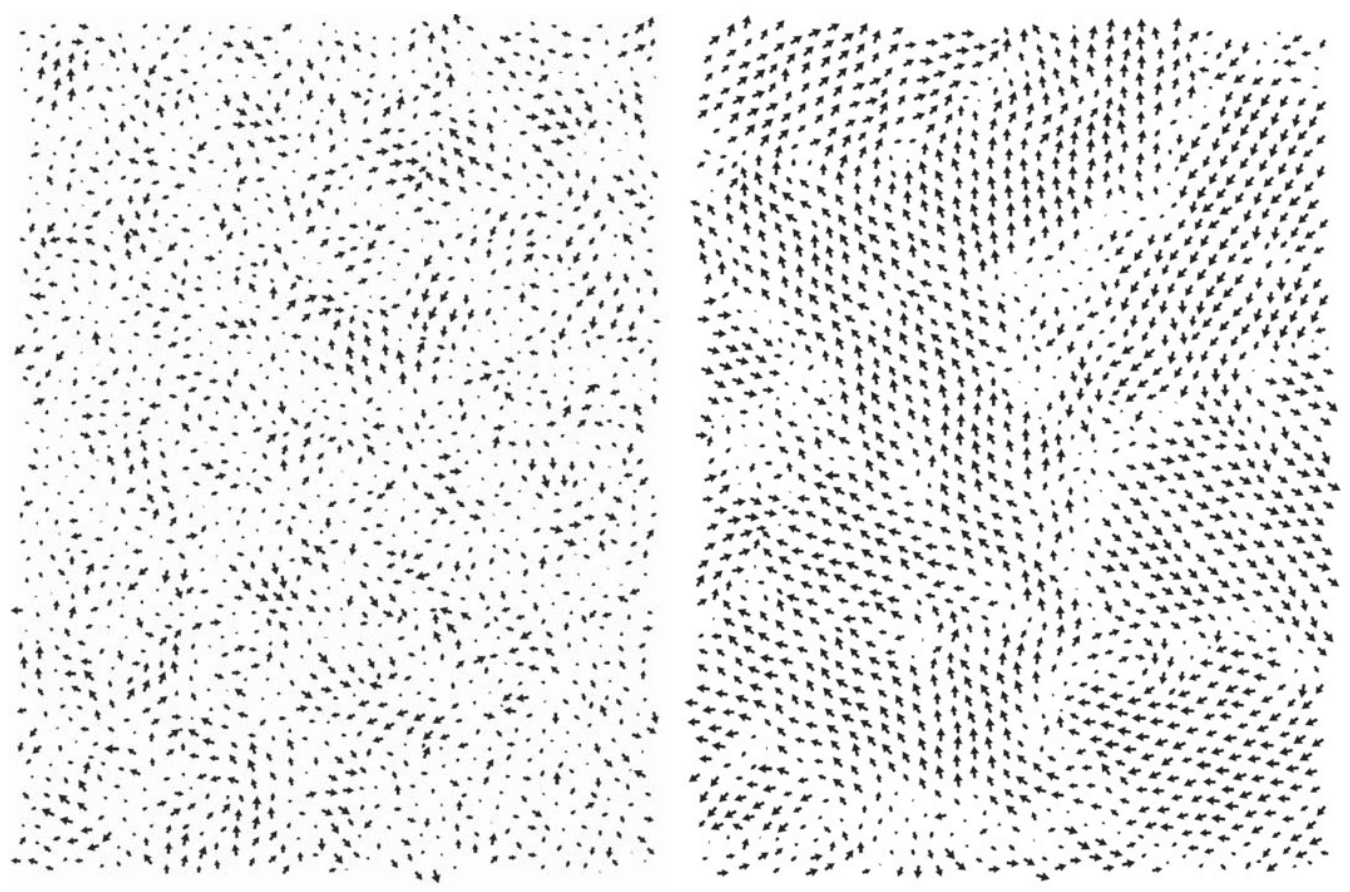

Figure 3. Cut-out of the director field of the particles based on the phase of the bond-order parameter. Left side: in the fluid phase $(\Gamma=20)$ the directors are randomly distributed and the magnitudes of the vectors $\propto m_{6}$ are small. Right side: polycrystalline phase, $2000 \mathrm{~s}$ after a quench from $\Gamma=20$ to 150 . The directors are arranged in grains where they are highly correlated, separated by grain boundaries. $m_{6_{k}} \leqslant 1$ within the grains and $m_{6_{k}} \ll 1$ in the boundaries.

The second criterion to distinguish crystal-like particles from fluid ones is based on the local bond-order parameter which takes into account the sixfold symmetry of the hexagonal crystal [18]:

$$
\begin{gathered}
m_{6_{k}}=\left|\psi_{k}\right| \\
\psi_{k}=\psi\left(\overrightarrow{r_{k}}\right)={ }_{N_{j}}^{1} \sum_{j} \mathrm{e}^{\mathrm{i} 6 \theta_{j k}},
\end{gathered}
$$

where $\theta_{j k}$ is the angle of the bond between particle $k$ and one of its nearest-neighbour particle $j$ relative to a fixed reference axis. The sum is taken over all the $N_{j}$ nearest neighbours. $m_{6_{k}}$ can be considered as a measure quantifying the extent to which the bonds between particle $k$ and its neighbours have sixfold symmetry. $m_{6_{k}}$ is zero for perfect five- or sevenfold coordinated particles and equals 1 for perfect sixfold particles. A projection of $\psi_{k}$ onto the mean local orientation field given by the $N_{l}$ nearest neighbours of particle $k$ is defined as

$$
n_{6_{k}}=\left|\psi_{k}^{*} \frac{1}{N_{l}} \sum_{l} \psi_{l}\right| .
$$

$n_{6_{k}}$ takes the second shell into account and quantifies how good the orientation of particle $k$ fits into the local hexagonal symmetry which is defined by the $N_{l}$ neighbour particles. Since it is a projection one finds $n_{6_{k}} \leqslant m_{6_{k}}$, and furthermore $n_{6_{k}}+m_{6_{k}} \leqslant 2$. Figure 2 (right) shows the probability distribution in the $m_{6}-n_{6}$ plane. In [18] values of $m_{6}+$ $n_{6}>1$ (upper right corner) were used as an empirical criterion for crystal-like particles. In our quenched system, especially when the grains start to touch, this does not lead to well-defined crystallites. Since $n_{6_{k}}$ is based on the secondnearest-neighbour shell, the thickness of grain boundaries is overestimated this way [19].

Both methods assign a scalar value to each particle and, in principle, a threshold of it should discriminate crystalline particles from fluid ones. But even when combining both criteria we were not able to distinguish undoubtedly between crystalline and fluid particles. This is unlike in $3 \mathrm{D}$, where a scalar value is sufficient to distinguish locally both phases $[14,15]$ because in 2D the local symmetry of the fluid is often very similar to the crystalline one. Even deep inside the fluid phase most particles have six nearest neighbours in 2D.

Therefore we developed a new criterion still based on the local bond-order parameter but taking into account the orientation relative to an arbitrary but fixed reference axis. This is done by the phase of the complex number $\psi_{k}$. The orientational phase of nearest-neighbour particles varies only slightly in a crystallite and the magnitudes of $\psi_{k}$ have values close to $1 . m_{6_{k}}$ is needed as a second criterion to distinguish five- or sevenfold coordinated colloids which may have accidentally the same bond orientation but should, of course, not be assigned to a crystal grain. In the liquid state, however, one finds randomly distributed phases/orientations and values of $m_{6_{k}}$ being less than 1 . Figure 3 shows this director field constructed of the local bond-order for each individual particle. The length of the vectors corresponds to the magnitude $m_{6_{k}}$ of the complex local bond-order parameter whereas the orientation equals an angle $\Theta_{k}$ (with respect to the $x$ axis) which is given by the phase of the complex number 


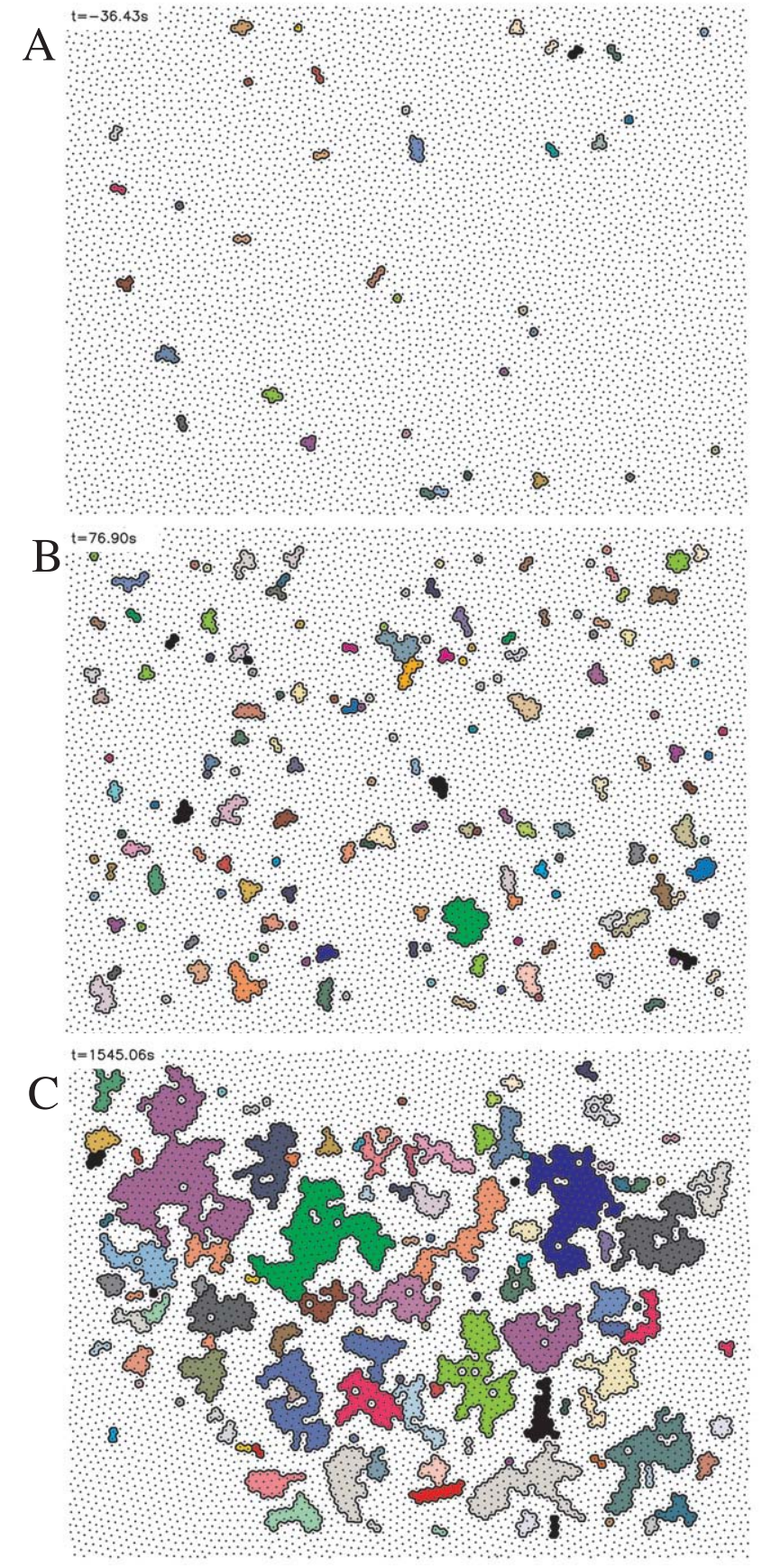

Figure 4. The Voronoi cells for particles recognized to be crystal-like are shaded. Colloids belonging to the same grain have the same shade. (A) In the fluid phase $\Gamma=20$ some small clusters do appear but vanish within a few seconds. (B) Crystal grains of the 2D sample $77 \mathrm{~s}$ after the quench from $\Gamma=20$ to 80 . (C) Same area $1545 \mathrm{~s}$ after the quench - the grains touch each other (only grains which are completely in the field of view are plotted).

$\left(1 / N_{j} \sum_{j} \mathrm{e}^{\mathrm{i} 6 \theta_{j k}}\right)$ with respect to the Re axis in the complex plane.

\section{Formation of crystallites}

To identify crystalline particles we apply three criteria. In a first step, neighbouring particles are selected only if the

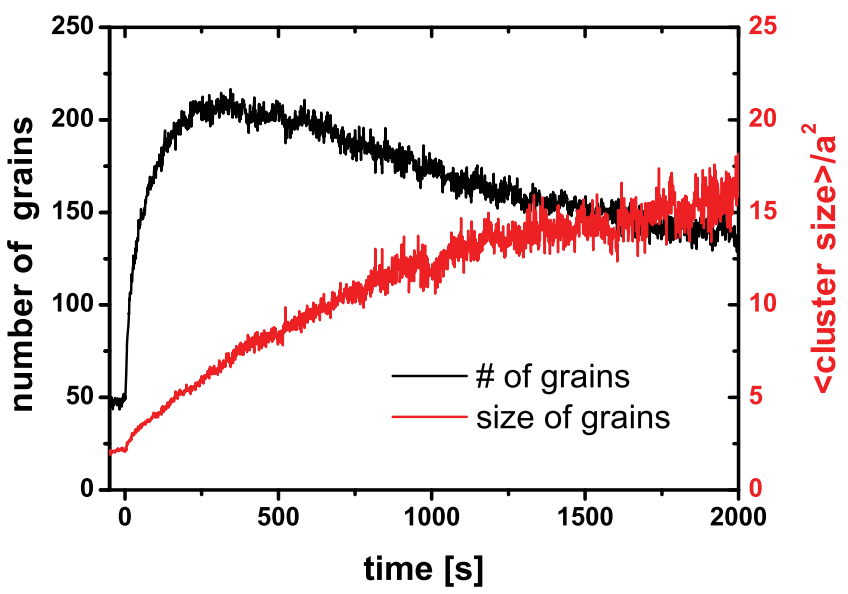

Figure 5. Number and size of crystallites as a function of time. $250 \mathrm{~s}$ after the quench from $\Gamma=20$ to 80 , the number of nuclei decreases again. This marks the time period, where the grains start to merge.

magnitudes of their local bond-order parameter $m_{6}$ exceed a certain threshold $m_{\mathrm{T}}$ and the phase difference $\Delta \Theta=\left|\Theta_{i}-\Theta_{j}\right|$ is smaller than $\Theta_{\mathrm{T}}$. These thresholds were chosen to be $m_{\mathrm{T}}=0.7$ and $\Theta_{\mathrm{T}}=15^{\circ}$ and are motivated in the following way. Both criteria were sharpened as long as a defect-free monocrystalline area, which was tempered over several days, is still recognized as one single crystal. When they are fulfilled we investigate whether those particles have at least three adjacent particles which also fulfil the above criteria. In that case only, particles are considered to be crystal-like and again, if they have a phase deviation less than $\Theta_{\mathrm{T}}$, they belong to the same grain. This is indicated in figure 4 for three different time steps before and after the quench. Random structural fluctuations may lead to crystal-like particles or small clusters even in the liquid state. They contain only a few particles and vanish within a few seconds. In a first time period after a temperature quench we observe the nucleation of crystal clusters. Some of the nuclei dissolve again in the fluid phase but others start to grow. As illustrated in figure 5 between $0 \mathrm{~s}<\tau<250 \mathrm{~s}$ the number and the size of the crystallites increase. This period is limited by a second one $\tau>250 \mathrm{~s}$, where the crystallites start to touch. Then a ripening process starts where the particles are reorganized and the large crystallites grow at the expense of the small ones. The number of crystallites decreases in this stage and their size increases at a lower rate.

\section{Conclusion and perspective}

We developed a criterion which unambiguously distinguishes between crystal-and fluid-like particles in a 2D colloidal ensemble. It is based on the phase of the complex local bond-order parameter and allows us to determine the size of crystallites after a temperature quench as a function of time. Further work will be to increase the time resolution in the first period after the quench to analyse the probabilities for growth and shrinkage of the grains. Thinking in terms of critical nuclei with favourable bulk and adversarial surface energies along the line of [14], the ratio of 2D bulk (= area) 
versus 2D surface (= borderline) energy is expected to be smaller compared to the 3D case, which would lead to smaller critical nuclei. While the system melts and crystallizes with two continuous phase transitions if it is always in thermal equilibrium [13], the scenario changes completely if the system is out of equilibrium-no indications of the hexatic phase, as an intermediate anisotropic fluid between isotropic fluid and crystalline phase, are observable.

\section{Acknowledgments}

We acknowledge fruitful discussions with Urs Gasser and Florian Ebert. This work was supported by the DFG (Deutsche Forschungsgemeinschaft) in the frame of SFB-513 project B6 and SFB-TR6 project C2.

\section{References}

[1] Kosterlitz J M and Thouless D J 1972 J. Phys. C: Solid State Phys. 5124

[2] Kosterlitz J M and Thouless D J 1973 J. Phys. C: Solid State Phys. 61181
[3] Chui S 1983 Phys. Rev. B 28178

[4] Glaser M and Clark N 1993 Adv. Chem. Phys. 83543

[5] Halperin B I and Nelson D R 1978 Phys. Rev. Lett. 41121

[6] Nelson D R and Halperin B I 1979 Phys. Rev. B. 192457

[7] Murray C A and Van Winkle D H 1987 Phys. Rev. Lett. 581200

[8] Tang Y, Armstrong A, Mockler R and O’Sullivan W 1989 Phys. Rev. Lett. 622401

[9] Kusner R E, Mann J A, Kerins J and Dahm A J 1994 Phys. Rev. Lett. 733113

[10] Marcus A H and Rice S A 1996 Phys. Rev. Lett. 772577

[11] Zahn K, Lenke R and Maret G 1999 Phys. Rev. Lett. 822721

[12] Grünberg H H v, Keim P, Zahn K and Maret G 2004 Phys. Rev. Lett. 93255703

[13] Keim P, Maret G and Grünberg H H v 2007 Phys. Rev. E 75031402

[14] Gasser U, Weeks E R, Schofield A, Pusey P N and Weitz D A 2001 Science 292258

[15] Gasser U, Schofield A and Weitz D A 2003 J. Phys.: Condens. Matter 15375

[16] Moucka F and Nezbeda I 2005 Phys. Rev. Lett. 94040601

[17] Reis P M, Ingale R A and Shattuck M D 2006 Phys. Rev. Lett. 96258001

[18] Larsen A E and Grier D G 1996 Phys. Rev. Lett. 763862

[19] Dillmann P Dissertation in preparation 